\title{
Les Arabes chrétiens, miroirs des échecs politiques du monde arabe
}

\section{Pierre Blanc}

Enseignant-chercheur à Bordeaux (Sciences po et Sciences agro), rédacteur en chef de Confluences Méditerranée.

L'année 2015 a entre autres été marquée par le centenaire du génocide des Arméniens et des Assyriens. Partout où s'est établie leur diaspora, notamment en France, des colloques et diverses commémorations ont été organisés pour se souvenir de ces massacres sciemment organisés par le triumvirat jeune-turc (Ismaïl Enver, Talaat Pacha et Djemal Pacha) après ceux perpétrés par le sultan Abdulhamid entre 1895 et 1896. Dans un moment de grande déliquescence de l'Empire ottoman, ces dirigeants ultranationalistes voyaient comme une évidence l'expulsion de ces éléments chrétiens arméniens et assyriens en vue de purifier le corps politique et social d'une nation turque de leur point de vue en danger. Qui plus est, parce qu'elles étaient établies surtout dans les provinces orientales de l'Empire ottoman, ces populations étaient accusées d'être la cinquième colonne d'une Russie orthodoxe poussée par son tropisme vers le sud et ses mers chaudes.

Cent ans après, le monde assiste dans la région à une même politique d'épuration sinon ethnique, du moins religieuse. Il n'est plus le fait d'ultranationalistes turcs mais des islamistes radicaux, ou néosalafistes messianiques, de l'État islamique et, sans les cibler exclusivement, ce nouvel épisode tragique affecte encore des chrétiens. Remplaçant l'État islamique d'Irak et du Levant (EIIL), l'État islamique est proclamé le 29 juin 2014 par Abou Bakr al-Baghdadi (de son vrai nom Ibrahim al-Badri) à Mossoul, l'ancienne Ninive biblique. Dans cette ville du nord de l'Irak, les populations non sunnites (chrétiennes et yézidies) doivent alors choisir entre la conversion, le «glaive » ou l'impôt. Tout cela dans un contexte de violences et d'islamisation forcée de leurs lieux de culte. Au début du mois d'août, le front que tiennent alors les militaires du gouvernement régional kurde (GRK) au nord de la ville finit par céder sous les coups de boutoir de Daech qui s'empare de la plaine de Ninive et de la ville de Qaraqosh. Comme à Mossoul, les populations chrétiennes doivent se soumettre par la conversion ou l'impôt quand elles ne sont pas exterminées. Anticipant cela, la plupart ont quitté cette région pour se réfugier dans le territoire du GRK. En février 2015, le même scénario se produit dans les villages ruraux 
situés le long du Khabour à proximité de la ville syrienne d'Hassaké. Ces villages sont conquis par Daech et, quand ils n'ont pas réussi à quitter leurs maisons, leurs habitants sont enlevés en nombre sans que l'on sache trop leur destination. S'ils sont soumis à cette politique d'épuration, c'est parce que ces villages sont essentiellement peuplés de paysans assyriens venus le long de la rivière Khabour en 1933, après qu'ils furent persécutés en Irak ${ }^{1}$.

Ces récents développements soulignent avec force la violence d'une représentation politique qui fait, dans le cas présent, de l'appartenance à l'islam - revu à la grille salafiste djihadiste - le critère d'élection exclusif de la «construction étatique ». Au-delà de cette représentation qui vise à exclure, ce phénomène marque de façon brutale l'échec des élites politiques du monde arabe dont l'impéritie et l'incurie de ses pouvoirs ont préparé le terreau d'une telle réponse idéologique mortifère ; échec aussi pour ces pouvoirs de n'avoir pas vraiment réussi à intégrer la diversité ethnoreligieuse dans leurs constructions politiques contemporaines [Otayek, 2013]. Revenir sur la situation des Arabes chrétiens apparaît donc nécessaire mais autrement qu'en recourant à une grille identitaire utilisée parfois en Europe. En effet, en suggérant par exemple qu'ils seraient des Arabes à privilégier dans le cadre de l'accueil des réfugiés - car ils seraient plus compatibles avec les Européens que les Arabes musulmans -, certains discours demeurent à tout le moins délétères. Cette tendance à la distinction a pu aussi s'insinuer dans la recherche, certes sous une forme moins grossière. Ainsi, l'intellectuel Hisham Sharabi introduisit une dichotomie entre les intellectuels arabes chrétiens, qui auraient été épris d'idéal laïque parce que tournés vers l'Europe, et les Arabes musulmans qui seraient restés contraints dans leur système de pensée par un enfermement islamique. Comme le fait remarquer Georges Corm, il s'agissait en l'occurrence d'une «mésinterprétation du rôle des penseurs arabes chrétiens » [Corm, 2015].

Avec le souci d'éviter ce genre de dichotomie, un retour sur la situation des Arabes chrétiens paraît d'autant plus nécessaire que, en ce moment de grande violence, leur présence parmi leurs concitoyens arabes musulmans est menacée alors qu'elle signe la pluralité séculaire du monde arabe. Or, par-delà les vies qui sont en jeu, c'est toute leur contribution culturelle, sociale et politique aux sociétés arabes qui est en péril. Évoquons ici des figures aussi diverses que la chanteuse Fayrouz (Nouhad Haddad), le musicien Marcel Khalifé, les cinéastes Youssef Chahine, Elia Souleymane, Michel Kheifi, Nadine Labaki, l'intellectuel Edward Said, les

1 Les Anglais s'étaient appuyés sur eux lorsqu'ils occupaient l'Irak et les Kurdes leur firent payer cette forme d'allégeance. 
écrivains Émile Habibi et Amin Maalouf pour ne citer que quelques figures contemporaines parmi les plus connues. Citons aussi les grandes universités chrétiennes que sont l'Université américaine et l'université Saint-Joseph de Beyrouth, l'Université pontificale de Bethléem ou l'université Al-Hikma à Bagdad, nationalisée dans les années 1960. Ou encore la part active prise par les chrétiens dans la création et l'animation de grands journaux comme Al-Hilal, AlAhram en Égypte et An-Nahar au Liban. Enfin, et surtout, rappelons leur participation active à la renaissance arabe au tournant du XIX ${ }^{\mathrm{e}}$ siècle.

Nous ne parlerons pas ici du christianisme émergent au Maghreb du fait des poussées évangéliques récentes, ni de la présence chrétienne dans les pays du Golfe car elle concerne essentiellement des immigrés asiatiques. Nous évoquerons le Proche-Orient où le christianisme arabe est l'héritier d'une longue histoire, cependant que sa présence est aujourd'hui très menacée sous l'effet des rivalités qui s'y déchaînent et d'un islamisme radicalisé. C'est ainsi une lecture essentiellement géopolitique de la présence chrétienne qui est ici proposée avec, en ligne de mire, la question de sa participation politique.

\section{Une géographie très politique}

Dans cette région, c'est au Liban que se trouve de loin la plus grande proportion de chrétiens (environ $35 \%$ ), devant la Syrie où ils représentaient quelque $10 \%$ de la population avant la guerre civile et l'Égypte où, selon les chiffrages, ils seraient entre 6 et $12 \%$. Mais c'est dans ce pays qui compte 80 millions d'habitants que les chrétiens sont les plus nombreux. En Irak, ils ne seraient plus que 400000 , quand ils dépassaient encore le million avant l'intervention américaine en 2003. En Jordanie, leur nombre tourne autour de 200000 et en Palestine ils ne seraient plus que quelque 60000 tandis qu'en Israël leur nombre avoisinerait les 120000.

Divisés par des schismes à causalité géopolitique plus que théologique ${ }^{2}$, les chrétiens se trouvent donc assez répartis dans l'espace. La topographie des lieux, le degré supposé

2 Après le concile d'Éphèse en 431, le succès du diphysisme prêché par Nestorius, qui distingue les natures divine et humaine du Christ, s'explique par des facteurs géopolitiques.

En effet, la doctrine de Nestorius gagne alors la Mésopotamie où les rois perses sassanides, de religion zoroastrienne, y trouvent un motif de soustraire leurs sujets chrétiens à l'influence de leurs voisins byzantins. Cette Église nestorienne ou assyrienne de langue syriaque s'établit donc de l'Est turc à l'Iran actuel. Lors du concile de Chalcédoine en 451, les partisans du monophysisme insistent sur la nature divine du Christ. Plutôt égyptiens, syriens et arméniens, les partisans de cette doctrine, en adoptant une doctrine monophysite et une langue liturgique (égyptien, syriaque et arménien) différentes du grec, prennent leurs distances avec l'Église byzantine et, partant, avec l'Empire. À son tour l'Église syrienne ou jacobite se divise, un groupe réuni autour de l'ermite Maron dans la région d'Apamée en Syrie se séparant de l'Église mère pour fonder l'Église maronite qui ne veut pas rompre avec les conclusions de Chalcédoine, ce qui lui vaudra d'être pourchassée et obligée par les jacobites, puis par les 
d'hétérodoxie, l'éloignement des centres urbains très vite soumis par les musulmans ont contribué à dresser la carte de l'implantation des Arabes chrétiens au long des siècles. Longtemps, certaines des communautés chrétiennes se sont ainsi retrouvées dans les «montagnes refuges» [de Planhol, 1968] : c'est le cas du Mont Liban qui accueillait les « hétérodoxes » maronites, des montagnes du Qalamoun, de Sam'an et du Hauran syriens, des montagnes du nord de l'Irak qui abritèrent de nombreuses générations d'assyriens et de chaldéens ; d'autres vivaient sur les côtes du Liban, de Palestine et de Syrie, en particulier les orthodoxes qui avaient été moins persécutés que leurs coreligionnaires chrétiens parce qu'ils avaient été reliés à l'Empire byzantin longtemps dominant dans la région ; enfin, certains se sont accrochés à des lieux à forte signification religieuse pour eux (par exemple, en Israël/Palestine, Nazareth, Bethléem et Jérusalem où les chrétiens étaient majoritaires jusqu'en 1948). Les coptes, eux, sont demeurés principalement en Haute-Égypte où l'islamisation n'avait pas été totale. Cependant, aux $\mathrm{XIX}^{\mathrm{e}}$ et $\mathrm{XX}^{\mathrm{e}}$ siècles, cette répartition a évolué : même si les points d'ancrage évoqués ont subsisté, la tertiarisation des économies et, partant, l'urbanisation voire la littoralisation des populations ont fait de certaines villes des lieux de forte présence chrétienne (Lattaquié, Alep, Homs, Damas, Beyrouth notamment).

Par-delà ces évolutions de localisation opérées au $\mathrm{XX}^{\mathrm{e}}$ siècle, c'est la forte diminution de leur nombre qui est aussi à signaler : $24 \%$ de la population totale du bilad al-cham (Syrie, Liban, Palestine, Jordanie) était chrétienne en 1914, contre moins de $10 \%$ aujourd'hui [Courbage et Fargue, 1992]. Cette diminution est liée en partie au tassement de leur accroissement naturel intervenu avant celui de leurs compatriotes musulmans. La présence de missions chrétiennes dans cette région explique l'accès globalement plus rapide à l'éducation des chrétiens et ainsi la baisse précoce de leur fécondité. Pays le plus multiconfessionnel de la région, le Liban est particulièrement exemplaire de ce décalage dans les transitions démographiques [Courbage et Boustani, 2008]. Même s'il y a actuellement une convergence démographique des différentes communautés ${ }^{3}$, la représentation d'un dynamisme démographique islamique demeure encore

musulmans, de gagner le Liban, une « montagne refuge » qui accueillera d'autres minorités persécutées (les chiites et les druzes). Au cœur de ces Églises copte, syriaque et arménienne, certains renoueront avec Rome à la faveur de l'arrivée des missions à partir du XVII siècle, d'où la présence d'Arméniens, de coptes et de syriaques catholiques. Avec le grand schisme du XI ${ }^{\mathrm{e}}$ siècle, c'est un autre divorce de type politico-ecclésial qui se produit plus qu'une séparation profonde sur le plan doctrinal. Le patriarche de Constantinople resté uni au pape de Rome, malgré un éloignement géographique et linguistique, finit par se détacher de celui-ci à la faveur de la crise de 1054. Parmi les orthodoxes («fidèles à la doctrine »), certains, à leur tour, se rapprocheront de Rome à partir du XVIII siècle en devenant grecs-catholiques.

3 Dans leur article Courbage et Boustani font état d'un indice de fécondité de 2,1 chez les chiites, de 1,76 chez les sunnites et de 1,61 chez les maronites alors qu'en 1971 celui des premiers était le double de celui des derniers. 
très prégnante chez certains chrétiens qui s'en inquiètent d'autant plus que certains secteurs de l'islam se trouvent actuellement dans une phase de crispation ou de radicalisation à leur endroit. Si le décalage démographique a contribué à diminuer la proportion de chrétiens dans la région, aujourd'hui c'est l'exode qui en est la cause majeure, même s'il affecte également les autres communautés. Les réseaux diasporiques anciens des Arabes chrétiens, particulièrement en Amérique du Sud et du Nord, en Europe et en Australie, facilitent ces départs.

Après avoir connu plusieurs vagues dans l'histoire contemporaine, cet exode des chrétiens est lié à l'état d'insécurité politique et économique qui affecte la région depuis la Seconde Guerre mondiale. Bien avant la guerre civile en Syrie et en Irak, ces flux étaient déjà très actifs même s'ils le sont encore plus du fait de l'intensification de la conflictualité et de la montée en puissance d'un islamisme radical tel que l'incarne l'État islamique.

\section{La montée en puissance d'une représentation qui exclut}

Ledit État islamique est l'avatar le plus brutal d'une idéologie islamiste qui n'a cessé de dériver vers le radicalisme depuis plus d'un siècle qu'elle a commencé à s'affirmer au Moyen-Orient dans un contexte de décomposition de l'influence ottomane. Certes, le discours de cette mouvance ne s'est pas construit par rapport aux chrétiens du monde arabe. Il s'agit avant tout d'une vision totalisante de la société et de l'État islamiques avec des variations très fortes au sein de cette mouvance, d'une personnalité à l'autre et d'un mouvement à l'autre. Et c'est dans cette vision totalisante, nourrie d'une lecture très littéraliste des sources de l'islam, qu'apparaissent en particulier des considérations sur les chrétiens comme sur les juifs.

Dans le panthéon des théoriciens de l'islamisme radical se détachent certaines figures dont les écrits sont porteurs de représentations radicales et hostiles envers les autres religions, en sachant que les épigones d'Abou Bakr al-Baghdadi, l'autoproclamé calife de l'État islamique, dépassent par leurs pratiques brutales les prescriptions de ces penseurs. Le plus ancien parmi eux est le jurisconsulte Ibn Taymiyya qui vécut au $\mathrm{XIV}^{\mathrm{e}}$ siècle, dans une période de conflit entre les Mamelouks musulmans et les Mongols, certes musulmans, mais comptant dans leurs armées des chrétiens (et qui de plus étaient alliés aux royaumes chrétiens). Dans son Livre du Glaive dégainé contre qui insulte le Messager, s’il épargne les moines car ils vivent reclus et sans influences, il cible surtout les « chefs de la mécréance »-prêtres ou autres - qui fourvoient les esprits qui les suivent. Mais sa pensée sur les chrétiens n'est que secondaire par rapport à bien d'autres aspects et diatribes contre les soufis et les chiites. Penseur du XIV ${ }^{\mathrm{e}}$, il garde pourtant une actualité chez les islamistes tout comme il a été un inspirateur central du mouvement 
wahhabite émergé au XVIII ${ }^{\mathrm{e}}$ siècle dans le Nadj et dont les chiites, nombreux dans la péninsule, ont été alors la cible religieuse.

C'est surtout au $\mathrm{XX}^{\mathrm{e}}$ siècle que la mouvance islamiste s'est enracinée dans le paysage moyenoriental et des circonstances sociopolitiques ont fait que son influence s'est répandue sur une partie croissante de l'opinion arabe. Au préalable, le Perse Jamal Eddine al-Afghani (18391897) qui produisit l'essentiel de son œuvre en Égypte et son disciple cairote Mohamed Abduh (1849-1905) avaient développé une pensée appelant à réhabiliter une certaine rationalité islamique, comme au temps des grands ancêtres (salaf), en vue de permettre aux Arabes de retrouver le lustre du passé après des siècles de tutelle. Mais, si cette pensée ne se voulait pas forcément exclusive du point de vue religieux, une première bifurcation plus radicale se produisit sans doute avec le Libanais Rachid Rida (1865-1935) qui, nostalgique du califat (supprimé en 1924) et plus perméable au wahhabisme de l'Arabie voisine, dénonça souvent les Européens et les Arabes chrétiens au mieux comme des rivaux, au pire comme des ennemis. Dans une Égypte dominée par les Britanniques, cette pensée inspira Hassan el-Banna, qui fonda les Frères musulmans en 1928. Cependant le djihad qu'il préconisait, au moins au début de l'histoire des Frères, devait surtout être dirigé contre l'autorité britannique. C'est son contemporain Sayyed Qotb qui approfondit la réflexion islamiste en la radi- calisant, notamment par rapport aux «mécréants » chrétiens. Pour cet intellectuel originaire de HauteÉgypte, l'expérience des États-Unis où il séjourna de 1948 à 1950 fut un catalyseur ou au moins un révélateur de son conservatisme [Qotb, 2000, p. 26-27]. À peine revenu de ce pays, il s'était engagé en 1952 dans l'association des Frères musulmans pour mener une lutte sans merci contre ce qu'il estimait être l' «esprit décadent» de l'Occident. Après une lune de miel entre le nouveau régime égyptien et l'association des Frères, la tentative d'assassinat contre Nasser attribuée à un Frère musulman réduisit considérablement l'espace politique pour les Frères qui furent alors fortement réprimés, à commencer par Sayyed Qotb, notamment à compter de 1954. Imprégné notamment des pensées d'Ibn Taymiyya, de Mohamed Ibn Abdel Wahhab et de Rachid Rida, c'est en prison qu'il rédigea l'essentiel de ses écrits. Dans son célèbre Jalons sur la route, il voulut démontrer la supériorité de l'islam et appelait à ne pas craindre le martyre pour faire advenir sa domination. Il allait même plus loin en considérant que le djihad ne devait pas concerner uniquement la «défense de la patrie de l'islam », autrement dit les pays de tradition islamique, car ce serait considérer que toute la terre n'avait pas à se soumettre à la souveraineté de Dieu. Du point de vue géopolitique, l'incidence est considérable puisque le domaine de l'islam - ^ar a/-M/arn - doit s'étendre à l'échelle planétaire. Aussi Sayyed Qotb 
appelait-il à la constitution d'une avant-garde éclairée en mesure de restaurer les valeurs de l'islam afin de les diffuser ensuite au reste de la terre pour que les hommes vivent dans un état de justice.

Les réflexions de Sayyed Qotb sur les chrétiens mais aussi les juifs sont sans doute les plus abondantes dans le champ islamiste. S'appuyant sur les écritures coraniques et devant les contradictions qui y apparaissent à propos des gens du Livre, il use de la règle de l'abrogeant et l'abrogé pour ne retenir que les sourates les plus coercitives [Carré, 2004] ${ }^{4}$. Par ailleurs, dans son ouvrage À l'ombre du Coran, il revient longuement sur ce qu'il considère comme un égarement des chrétiens sur le plan christologique et théologique. Il leur reproche d'avoir dévoyé la figure de Jésus en le divinisant, et en considérant d'ailleurs que seule la doctrine arienne, voulant que Jésus ne fût pas fils de Dieu, était pleinement musulmane. Il revient aussi sur le dogme de la Trinité pour le critiquer mais en le déformant. Il s'appuie également sur l'Évangile de Barnabé, un évangile apocryphe écrit vraisemblablement en milieu morisque plus de quatorze siècles après Jésus-Christ [Fierro, 2009], dans lequel celui-ci aurait annoncé la venue du Prophète. Mais il va plus loin en considérant que «c'est un droit et un devoir de combattre les non-convertis » [Carré, 2004, p. 261]. Malgré ses positions extrémistes, il laisse la possibilité aux chrétiens et aux juifs de vivre dans le domaine de l'islam, moyennant la conversion ou, à défaut, le paiement de la jizya. On est donc dans un statut de protégé (dhimma) mais ici il demeure très dévalué puisque les musulmans sont appelés à ne pas entrer en amitié avec les chrétiens et les juifs, au moins tant qu'ils ne se sont pas convertis à l'islam.

Si l'on insiste ici sur le corpus doctrinal de Sayyed Qotb, c'est parce qu'aujourd'hui beaucoup d'islamistes - qu'ils soient djihadistes ou non - se réfèrent à lui pour avoir appelé à la constitution d'une avant-garde à laquelle les plus radicaux s'identifient [Kepel et Milelli, 2005]. Ses idées empreintes de perspective historique, sa vie à l'ombre des geôles et sa pendaison par le régime nassérien en 1966 ont sans doute décuplé son emprise dans les générations d'Arabes, constatant toujours plus l'échec de leurs régimes politiques. Mais si ces idées ont prospéré, c'est également par un concours de circonstances bien connues. Les Frères furent en partie expulsés d'Égypte dans les années 1960 et l'Arabie saoudite salafiste, en quête d'antidote au panarabisme laïque, trouva en eux un allié précieux, car ils étaient rompus à l'organisation

4 Olivier Carré considère que les versets 29-35 de la sourate IX qui concerne les non- musulmans abrogent ceux plus favorables de la sourate $\mathrm{V}$ et du début de la sourate II. 
politique. Cette frérisation ${ }^{5}$ progressive des salafistes wahhabites et les moyens financiers considérables permettaient à Riyad d'endiguer les idéologies supposées laïques dans le monde arabe mais aussi au-delà comme en Afghanistan. Mais on sait aussi que ce salafisme frérisé devait produire plus d'extrémisme encore au sein d'un islamisme radical voué à s'épanouir dans un contexte de déréliction du monde arabe. Car cette radicalisation progressive de l'islamisme et surtout son succès doivent beaucoup à l'échec social, économique et politique des nationalistes arabes, à la question palestinienne vécue comme une humiliation par nombre d'Arabes, puis, plus tard, aux dérives américaines de l'administration de George W. Bush dont la guerre en Irak a pu ouvrir un nouveau front pour des djihadistes en quête de guerre sainte. Qui plus est, avec l'embrasement de la fitna musulmane entre chiites et sunnites, sur fond de rivalités aiguës entre Riyad et Téhéran, les djihadistes ont trouvé aussi un catalyseur de leur haine. Daech en est l'exemple flagrant, et ses agissements violents contre les chrétiens et d'autres sont le paroxysme d'une lente dérive idéologique islamiste dont la prééminence donnée à l'appartenance islamique dans les constructions politiques porte en elle le terreau de l'exclusion.

Évidemment, l'islamisme est loin de se réduire à cette pointe extrême du djihadisme salafiste : des formations islamo-nationalistes - Hamas palestinien, Hezbollah libanais, Wasat égyptien, etc. - sans visée internationaliste, contrairement aux djihadistes, entretiennent des relations à tout le moins complexes avec les minorités qui peuvent aller jusqu'à la coopération politique, comme dans le cas du Hezbollah qui est allié du Courant patriotique libre de Michel Aoun ( $c f$. infra). Dans ces cas-là, non seulement il n'y a pas recours à la violence, mais il n'est pas non plus question de revenir au choix entre la conversion et l'acquittement de la jizya en échange d'un devoir de protection (dhimma), tel qu'il avait été plus ou moins pratiqué en terre d'islam avant l'arrivée des Ottomans.

Par-delà ce large spectre des expressions politiques de la référence islamique, il y aussi ce qu'on pourrait qualifier d'islamisme du quotidien, difficile à vivre pour tous ceux qui n'embrassent pas l'islam comme pour ceux qui, tout en partageant cette foi, ne supportent pas les prescriptions littérales et décontextualisées. Même si elles ne participent pas forcément d'une posture intégriste, le retour (ou le supposé retour) de certaines normes vestimentaires islamiques peut être ainsi difficile à assumer pour des chrétiennes qui se sentent de plus en plus distinctes dans

5 J'emprunte cette expression à Stéphane Lacroix, spécialiste de l'islamisme. 
des sociétés majoritairement musulmanes. En retour, cet «islamisme au quotidien » qui s'accompagne de fait d'un certain contrôle social et d'un marquage de l'espace public contribue au repli communautaire quand ce n'est pas au départ de ceux qui sont étrangers à cette norme religieuse.

Cet « islamisme du quotidien » est alimenté par des prédicateurs dont la présence médiatique est évidente. Il en est ainsi de Youssef al-Qaradawi, qui fut le célèbre animateur de l'émission «La shari'a et la vie », très suivie sur Al Jazeera, et dont les positions sur les gens du Livre sont des plus conservatrices. En effet, tout en se désolidarisant de l'islamisme radical, ce prédicateur célèbre n'en souhaite pas moins un retour à la règle de la dhimma [Seniguer, 2015].

\section{L'éviction des représentations politiques du dépassement}

Cette affirmation islamiste sous des formes très diverses explique la décrue de la présence chrétienne au Proche-Orient, sans pour autant en être la seule raison. Elle marque aussi le reflux de certaines idéologies politiques auxquelles s'étaient identifiés les chrétiens et qui, par-delà leurs diverses orientations (nationalisme, socialisme, etc.), avaient toutes pour particularité de dépasser un certain enfermement religieux. Cet affaissement idéologique a pu s'observer en particulier au moment des soulèvements du début de la décennie 2010, dans le monde arabe en général et au Proche-Orient en particulier. Les mobilisations en Égypte notamment ont montré l'insuffisance de formulations et d'organisations politiques en dehors des courants islamistes qui, du fait de leur niveau d'organisation, de leur cohésion et de leur discours empreint de sacralité, ont profité des événements plus qu'ils ne les ont provoqués.

Étant donné la vigueur de ces formulations islamistes qui excluent les minorités religieuses, d'une part, et l'effondrement des idéologies du dépassement communautaire, d'autre part, les Arabes chrétiens se sentent de plus en plus marginalisés sur le plan politique, ce qui les contraint à la communautarisation quand ce n'est pas au départ. Ils ont été pourtant en première ligne des combats politiques au $\mathrm{XX}^{\mathrm{e}}$ siècle. Mais, comme le faisait remarquer Carole Dagher,

ayant servi sur tous les fronts et brandi toutes les bannières idéologiques possibles et imaginables, les chrétiens d'Orient se retrouvent au bout du compte, [...] à bout de souffle, ayant perdu leurs illusions, impuissants et convaincus que quelle que soit la doctrine politique qu'ils embrasseraient, ils seraient toujours perdants. Ils sont fatigués d'avoir à prouver régulièrement leur loyauté à un environnement qui n'arrête pas de douter d'eux, fatigués d'être des Sisyphe modernes et d'avoir à grimper la montagne en poussant inlassablement le rocher jusqu'au sommet [Dagher, 2002, p. 33]. 
Cet engagement politique des Arabes chrétiens remonte à la fin du $\mathrm{XIX}^{\mathrm{e}}$ siècle lorsque la fragilisation de l'Empire ottoman, d'une part, et l'affirmation de l'Égypte, d'autre part, ont donné lieu à la renaissance arabe (nahda) après des siècles de tutelles. C'est dans ce contexte que des intellectuels arabes, souvent chrétiens originaires de Syrie et du Liban, promurent l'idée d'un nationalisme arabe dont l'expression appelait avec plus ou moins de vigueur une certaine rupture avec l'Empire ottoman qui gardait la mainmise sur le califat. À côté d'intellectuels musulmans dont Abd al-Rahman al-Kawakibi (1849-1902), les chrétiens Boutros al-Boustani (1819-1882), Yacoub Sarraf (1852-1927), Adib Ishaq (1856-1885), Faris al-Chidiaq (18041887), Farah Antoun (1874-1922) et Jorji Zaydan (18611914) furent de grandes figures de proue de ce mouvement plus porté à célébrer la nation arabe qu'une nation islamique réunie autour du siège califal d'Istanbul. Au-delà de leur projet commun d'émancipation de la tutelle turque, ces intellectuels exaltaient une certaine arabité à même de dépasser le clivage confessionnel. À noter que cette implication des chrétiens dans la renaissance arabe est liée à leur niveau d'éducation globalement plus élevé, du fait de la présence d'un système scolaire importé dès le XIX ${ }^{\mathrm{e}}$ siècle, dans les régions chrétiennes du Levant, par les missionnaires européens. Cependant, étant donné leur critique en creux du pouvoir ottoman, certains de ces intellectuels furent dans l'obligation de se réfugier en Égypte, un pays certes dégagé de l'emprise ottomane mais sous tutelle britannique. Cette immigration syro-libanaise participa ainsi à l'essor culturel (presse, théâtre, roman, poésie, cinéma) en Égypte, véritable foyer de la renaissance arabe.

Sur le plan politique, ce nationalisme arabe a connu plusieurs vagues au $\mathrm{XX}^{\mathrm{e}}$ siècle et les chrétiens y ont pris une large part. Parangon du panarabisme, le nassérisme ne fut pas celui qui en accueillit le plus parmi eux, ne serait-ce que parce que l'institution militaire sur laquelle il s'appuyait comptait très peu de chrétiens. C'est surtout le Parti socialiste de la renaissance arabe (Baath), encore au pouvoir aujourd'hui en Syrie, qui allait offrir un toit politique à de nombreux chrétiens. Fondé en 1947 notamment par le grec-orthodoxe Michel Aflak, celui-ci développa une philosophie politique basée sur le triptyque unité, liberté et socialisme. Inspiré du personnalisme chrétien d'Emmanuel Mounier, Michel Aflak insistait sur la nation comme « cadre concret et historique du développement des potentialités de l'homme. L'humanisme ne se réalise que par le chemin médiat de la nation » [Khader, 1984, p. 132]. Chaque nation, en vivant ou en renaissant, avait donc pour vocation de faire vivre la liberté humaine plus concrètement qu'en affirmant un vague universalisme. Avec son identité propre fondée notamment sur la proximité linguistique de ses composantes, la nation arabe devait ainsi se 
rassembler. Et pour atteindre cet épanouissement de la personne au travers de la libération de la nation arabe, il prônait un socialisme capable de reprendre le contrôle d'une richesse confisquée par des élites politiques adoubées par des puissances tutélaires, qui s'étaient elles aussi emparées d'une partie du capital productif arabe. Le parti Baath réussit à s'implanter dans le paysage politique syrien très agité des années 1950 avant de prendre le pouvoir en 1963 et de basculer vers l'autoritarisme. Ce durcissement coïncidait avec la prise du parti par des alaouites (minoritaires) qui se vengeaient ainsi de la discrimination dont ils furent l'objet pendant des siècles. Ils commencèrent alors à constituer le noyau dur d'un parti- État qui prenait soin, quand même, de ne pas se couper des autres communautés du pays, en premier lieu des chrétiens qui s'étaient engagés dans ce parti laïque dès sa création.

Cette trajectoire du Baath fut assez similaire en Irak puisque, en dépit de ses accents laïques, il se resserra autour des sunnites (minoritaires) qui dominaient le pays depuis l'indépendance. Là aussi, avant de disparaître en 2003, ce parti-État s'attacha à ne pas se couper des autres minorités notamment chrétiennes (pensons à Tarek Aziz ministre des Affaires étrangères de Saddam Hussein), mais en laissant la direction du parti aux sunnites de la région de Tikrit d'où Saddam Hussein était originaire.

Moins influente mais significative de l'engagement des chrétiens dans les formations laïques nationales fut la mouvance pansyrienne, qui affirmait l'existence d'une communauté culturelle entre peuples de la Syrie historique, y compris donc le Liban, la Palestine et la Cilicie. Apparue sur fond de déliquescence de l'Empire ottoman, cette mouvance chercha à mettre en exergue le bien-fondé de cette Syrie historique. Elle fut portée à la veille de la création des États en 1920 par Chucri Ghanem, un maronite beyrouthin, George Samné, un grec-catholique de Damas, et Jamil Mardam Bey, un sunnite damascène. Cependant la création du Grand Liban (1920) fit perdre une partie de ses illusions à cette mouvance. Elle donna quand même naissance au Parti populaire syrien (PPS) créé par le grec-orthodoxe Antoun Saadé (1904-1949) qui n'avait de cesse de mettre en lumière l'unité géohistorique et culturelle de l'Irak, de la Syrie, de la Palestine, du Liban et de Chypre. Pour le PPS, le fond araméen et syriaque, lui-même antérieur dans la région au fond arabe, constituait le soubassement de cette unité. Comme le panarabisme, le pansyria- nisme ne faisait donc pas coïncider la nation avec l'oumma islamique, et ses accents demeuraient très laïques, ce qui explique que ce courant ait accueilli nombre de chrétiens ou des militants issus des confessions minoritaires (chiites, druzes, alaouites notamment). Actuellement, le Parti social national syrien (PSNS), héritier du PPS, ne compte qu'un organe au Liban où il se retrouve au cœur de la coalition dite du 8 mars, réunissant les formations 
chiites du Hezbollah et d'Amal ainsi que le Courant patriotique libre (CPL) du chrétien Michel Aoun.

Des nationalismes moins ambitieux dans leurs revendications territoriales, ont aussi accueilli moult chrétiens. Tel fut le cas du parti Wafd en Égypte qui s'engagea à ses débuts dans le refus de la tutelle britannique. Prenant la croix et le croissant comme symboles et appelant à la reconnaissance d'une seule citoyenneté égyptienne, il n'est guère étonnant qu'il ait accueilli beaucoup de coptes. Cependant, malgré sa légitimité historique, ce parti a été dissous par Nasser avant d'être autorisé par Sadate en 1978 mais maintenu à la périphérie d'un échiquier politique très contrôlé. Aujourd'hui, le néo-Wafd ne compte pas vraiment dans l'arène politique même si ses drapeaux étaient présents sur la place Tahrir lors de la révolution de 2011.

De son côté, le nationalisme libanais porta la création d'un État libanais à la conférence de Paris en 1919 contre les partisans de la Grande Syrie ou ceux de l'unité arabe. Dans le cadre de la Revue phénicienne, des intellectuels maronites ont fait émerger cette représentation d'une nation libanaise. Ils produisirent un récit national empruntant à des temporalités différentes (longue, avec l'évocation de la Phénicie, et plus courte, avec l'émirat de la Montagne sous les Ottomans) et insistant sur la mosaïque identitaire du proto-Liban. Ce nationalisme n'était donc pas confessionnel dans sa formulation et il a d'ailleurs été porté également par des Libanais musulmans. La lutte contre la puissance mandataire française se situa dans cette affirmation nationale, portée notamment par le Bloc constitutionnel du maronite Béchara el-Khoury et du sunnite Riadh el-Solh, qui prirent la tête du nouvel État indépendant en 1943. Cependant, ce nationalisme a échoué en tant qu'idéologie transcommunautaire car il s'est brisé sur les logiques confessionnelles du pays. Dès les années 1930, le Liban avait vu émerger le nationalisme beaucoup plus confessionnel des phalangistes, qu'on a pu qualifier de «maroni- tisme politique » tant il visait moins la transcendance des clivages religieux que la suprématie de la communauté maronite sur le reste de la société libanaise. Mais ce n'est qu'avec les guerres du Liban (1975-1990) que cette formulation très communautaire du libanisme politique s'exprima avec force, du fait de la présence des Palestiniens que les phalangistes et diverses milices chrétiennes percevaient comme une menace pour leur État [Faddoul, 2007]. La guerre civile consacra la segmentation communautaire de l'arène politique et, depuis la fin du conflit, seules quelques formations marginales (PSNS, parti communiste, gauche démocratique) gardent une composition pluricommunautaire. En dépit de cette communautarisation idéologique, les réflexions riches de Youakim Moubarak, Grégoire Haddad, George Khodr et Michel Hayek, 
tous prêtres libanais, demeurent comme une ressource inépuisable pour penser le dépassement communautaire [Fleyfel, 2011].

L'autre combat strictement national où les chrétiens se sont beaucoup engagés est celui de la Palestine. Ils s'impliquèrent d'abord dans l'association islamo- chrétienne créée dès 1918 ou dans la presse comme première réponse «nationale » au sionisme. Dépassant les clivages religieux, l'idée d'une nation palestinienne reçut par la suite un large écho chez les Palestiniens chrétiens dont l'insertion universitaire, culturelle et économique leur assurait un rayonnement réel. Mais la guerre de 1948 en poussa beaucoup à quitter leurs maisons et leurs propriétés en Galilée, dans la région de Lod et ailleurs. Cette catastrophe (nakba) et la guerre des Six Jours en 1967 renforcèrent le nationalisme palestinien et certains Palestiniens chrétiens prirent la tête de formations politiques. C'est le cas de George Habache et Nayef Hawatmeh qui se retrouvèrent au sein du Front de libération nationale de la Palestine (FPLP) créé en 1967, avant que le second ne fît sécession en fondant le Front démocratique de libération de la Palestine (FDLP). Moins connu mais très proche de Yasser Arafat, le père Ibrahim Ayad, partisan de la théologie de la libération, appartint au comité exécutif de l'OLP tout comme Elia Khoury, un évêque anglican. Faut-il aussi évoquer Hilarion Capucci qui fut un évêque très actif au point d'être emprisonné par Israël plusieurs années durant ?

Cependant, miné par l'occupation israélienne et la montée en puissance du Hamas, force est d'admettre que le nationalisme palestinien, dans sa version laïque, a perdu de sa vigueur politique.

Si beaucoup de ces nationalismes transcommunautaires ont échoué, la mouvance socialiste s'est, elle, totalement effondrée. En mettant en valeur les solidarités de classe plutôt que les assignations communautaires, ce courant idéologique constituait un vecteur de dépassement de l'oumma islamique pour de nombreux chrétiens arabes du Levant et d'Égypte ainsi que pour d'autres groupes minoritaires (alaouites, druzes, etc.). De son côté, Moscou savait également jouer l'alliance orthodoxe pour s'assurer un certain écho chez les grecs-orthodoxes, qui euxmêmes étaient souvent plus éloignés de l'Occident que d'autres communautés protégées par la France ou l'Angleterre.

\section{Re-communautarisation et exode : les tendances actuelles}

L'effondrement des idéologies du dépassement communautaire et la montée en puissance de l'islamisme entravent l'insertion politique des Arabes chrétiens qui n'a de fait jamais été vraiment totale. Du fait même que l'islam est resté la religion officielle dans bien des États, à 
l'exception du Liban, les chrétiens, même en étant actifs dans le champ politique, sont restés en effet éloignés de la fonction suprême présidentielle. Mais aujourd'hui c'est la question même de leur présence qui se pose, au moins dans certains pays. Actuellement se dessine une sorte de gradient des situations, de la plus difficile à la plus enviable.

C'est en Syrie et en Irak que leur situation est actuellement la plus délicate. Avant la guerre civile, les chrétiens de Syrie avaient misé, pour se protéger, sur une forme de loyauté par rapport au régime baathiste - au moins les dignitaires religieux et quelques personnalités laïques liées au régime - tandis que quelques opposants notoires avaient pris leurs distances (Michel Kilo, Anouar al-Bounni). La guerre civile a bouleversé cet équilibre précaire : après s'être pour certains engagés dans le soulèvement du début 2011, les chrétiens des agglomérations de Homs puis d'Alep notamment se sont retrouvés pris dans un conflit très violent. Dans les régions plus rurales du Qalamoun et de la Djéziré où ils étaient nombreux, l'arrivée du conflit a poussé nombre d'entre eux à l'exode. Désormais, entre la Charybde d'un régime brutal et la Scylla d'un islamisme djihadiste, beaucoup choisissent (si le verbe convient) d'en rester au premier qui se présente, non sans cynisme, comme le «protecteur» des minoritaires. Leur recommautarisation entamée avant la guerre dans un contexte de montée en puissance d'un «islamisme du quotidien »s'est ainsi accélérée. Mais beaucoup aussi, à l'instar de leurs compatriotes musulmans, décident de partir au Liban, en Jordanie ou ailleurs sans que l'on puisse en établir le nombre.

En Irak, les trajectoires sont un peu les mêmes, à ceci près que le régime autoritaire baathiste, qui était supposé les protéger, est tombé en 2003. Dans le chaos postconflit qui les a exposés directement ou indirectement aux attentats, beaucoup de chrétiens de Bagdad, Bassora et ailleurs ont trouvé refuge dans la région de Ninive à proximité du territoire administré par le gouvernement régional du Kurdistan (GRK). On sait ce qu'il est advenu ensuite avec l'offensive de l'État islamique dans ces régions au cours de l'été 2014. Beaucoup avaient aussi émigré en Jordanie, au Liban et en Syrie. Mais pour ces derniers arrivés dans les zones assez fortement chrétiennes de Damas (Jeramana, Saydanaya, Kachkoul), d'Alep et d'Hassaké, le sort s'est de nouveau acharné sur eux avec le déclenchement de la guerre civile.

En Irak comme en Syrie, certains chrétiens ont créé des milices de défense contre Daech comme Dwekh Nawsha («Futurs martyrs » en araméen) dans la région de Ninive, Suatoro dans la région d'Hassaké et Qamishli, le Conseil militaire syrien proche des Kurdes de l'Unité de défense populaire (YPG), mais ces engagements sont sans effets réels et peuvent les isoler davantage. Ceci leur vaut d'être parfois critiqués par les autorités religieuses qui préfèrent une 
intervention de la communauté internationale ou un engagement de ces volontaires dans les armées irakienne ou kurde, plutôt que dans une milice communautaire.

En Égypte, les coptes ont, eux aussi, été les témoins d'un soulèvement en 2011. Au préalable, un attentat les avait fortement touchés à Alexandrie lors des célébrations de Noël 2010, provoquant des mobilisations importantes qui figurent peut-être parmi les prodromes de la révolution de janvier-février. Cet attentat traduisait à la fois l'état d'insécurité et les dérives extrémistes du pays. Depuis les années 1970, la montée en puissance de l'islamisme dans le pays (avec la présence de groupes radicaux du type Jamaa islamiyya) les avait globalement portés à se contenter d'une sorte de sujétion au pouvoir tout en se communautarisant un peu plus [Guirguis, 2012]. La période du nationalisme égyptien dans sa version Wafd d'avant la Seconde Guerre mondiale était donc désormais très lointaine. Certes, des chrétiens avaient refusé ce rapport de sujétion et cette communautarisation ; c'est le cas de George Ishak, l'initiateur du mouvement Kifaya («ça suffit ») créé en 2005. Mais, dans le régime autoritaire et népotique de Moubarak, il s'agissait d'exceptions. Le renversement politique de l'année 2011, qui vit la multiplication des références à l'unité des chrétiens et des musulmans au fondement de l'Égypte, laissait espérer une certaine participation politique des coptes et une décommunautarisation de leur part. Deux événements les en ont rapidement dissuadés : d'une part, la multiplication des attentats anticoptes dont les révolutionnaires renvoyaient la responsabilité aux anciens du régime déchu et au régime transitoire perçu comme étant dans sa lignée ; d'autre part, les gains électoraux des islamistes entre 2011 et 2012. Ainsi, si certains coptes avaient été en première ligne des soulèvements de 2011, ils se retrouvaient marginalisés par la nouvelle donne politique qui voyait les Frères musulmans accéder au pouvoir. Faut-il s'étonner dès lors que certains de leurs leaders, au premier chef le pape copte Tawadros II, aient soutenu la manifestation du 30 juin 2013 et le renversement du pouvoir frériste ? Cependant, depuis cette reprise du pouvoir par les militaires, il s'agit davantage d'un retour à la sujétion ${ }^{6}$ (et sans doute à la communautarisation) qu'à l'exercice d'une citoyenneté pleine et entière.

En Israël et en Palestine, c'est une double pression du pouvoir israélien d'une part, et de l'islamisme d'autre part que les chrétiens subissent. En Israël, les Arabes israéliens, une appellation qui n'est guère appréciée d'eux-mêmes actuellement, tant elle nie leur identité palestinienne, ne sont pas des citoyens de plein droit : ils ont certes une représentation politique

6 Il n'est pas sûr que, dans le cadre de la nouvelle Constitution de 2014, l'attribution aux chrétiens d'un quota de vingt-quatre sièges au Parlement - un nombre jamais égalé au préalable - soit un vecteur suffisant d'affranchissement. 
à la Knesset mais les politiques différenciées dans le domaine social, éducatif et urbain ne les placent pas au même niveau de citoyenneté que les Israéliens juifs [Bendélac, 2008 ; Barrenada, 2013]. Les dotations aux écoles et aux villes arabes ne sont pas équivalentes aux structures juives ; pire, les vieilles villes de Saint-Jean-d'Acre et de Jaffa sont soumises à des politiques de judaïsation à l'instar de ce qui est très connu à Jérusalem. Plus spécifiquement, les chrétiens sont actuellement exposés au divide and rule des autorités israéliennes : en 2014, le Parlement a voté la possibilité pour les chrétiens d'effectuer le service national contrairement aux musulmans. Le porteur de ce projet de loi, le député Yariv Levin, du parti d'extrême droite Israel Beytenou, déclara ainsi sur le site web du quotidien israélien Maariv avant le vote du Parlement :

C'est un instant historique qui pourrait rééquilibrer l'État d'Israël et nous rapprocher des chrétiens, que je tiens à ne pas appeler arabes, car ils ne sont pas arabes. [...] Les chrétiens sont nos alliés naturels. Ils servent de contrepoids aux musulmans, qui veulent détruire l'État de l'intérieur. Les chrétiens sont aussi préoccupés par l'islam extrême, qui les exclut.

Jouer sur les peurs de l'islamisme est d'autant plus facile que les Palestiniens d'Israël sont eux aussi travaillés par ces courants. Dans la ville de Nazareth, le mouvement islamique a ainsi disposé une grande pancarte au ton comminatoire envers les chrétiens, leur rappelant de ne pas être trop audacieux sur leur credo ${ }^{7}$. Elle est installée juste devant la basilique de l'Annonciation, à l'endroit même où un projet de mosquée avait été approuvé par Israël à la fin des années 1990, mais auquel Yasser Arafat s'était opposé.

Dans les territoires occupés palestiniens, les chrétiens sont soumis à l'occupation et à la colonisation israéliennes. Certaines zones chrétiennes, comme la région de Bethléem avec les villages attenants de Beit Sahour et Beit Jala, demeurent des foyers de résistance civile très actifs. Avant les accords d'Oslo, la ville de Beit Sahour fut notamment en pointe dans le refus de payer l'impôt à l'occupant israélien, ce qui lui valut de subir des mesures de rétorsion massive. Actuellement, dans cette même région, les Palestiniens chrétiens de la vallée de Crémisan sont particulièrement menacés par les projets d'extension des colonies et du Mur. Quant aux quelque 3000 chrétiens de Gaza, ils vivent au rythme des guerres récurrentes entre le Hamas et Israël. Dans ce territoire paupérisé, dense et sans guère de perspectives, ils ont aussi

7 «Oh gens des écritures, les chrétiens ! Ne dépassez pas les limites dans votre religion. Ne dites rien d'autre que la vérité au sujet de Dieu. Le Christ Jésus, Fils de Marie, était seulement un messager de Dieu. [...] Ne croyez qu'en Dieu et en ses Messagers mais ne dites pas: "Trois divinités". Cessez ! Ce sera mieux pour vous. Dieu est l'unique Dieu. Sa Sainteté est bien au-delà d'avoir un Fils. » 
à vivre avec un islam qui se radicalise même si le patriarche Michel Sabah a pu déclarer en septembre 2014 que « le Hamas nous protège ${ }^{8}$ ».

À l'heure actuelle, les Palestiniens chrétiens demeurent très actifs dans le mouvement national. Il faut signaler notamment la mobilisation active de religieux et de laïcs dans le mouvement non-violent Kayros, inspiré de la théologie de la libération, qui relaie les appels à la campagne BDS (Boycott, Désinvestissement, Sanctions) contre Israël. Dans leur démarche, ils se sentent soutenus par l'accord récent entre l'État de Palestine et le Vatican ${ }^{9}$. Faut-il dès lors interpréter le ciblage des lieux saints du christianisme en Israël par certains mouvements de colons comme une réponse à cet accord et à l'audace des chrétiens de Palestine ?

En Jordanie, la situation des chrétiens - qu'ils soient d'origine bédouine ou palestinienne demeure somme toute favorable au regard de celles précédemment décrites. Très actifs dans le champ académique, économique et médical, les chrétiens ne sont pas absents non plus du champ politique. Des ministres chrétiens participent au pouvoir mais dans l'ensemble leur insertion relève de la protection - avec des sièges de parlementaires qui leur sont dévolus - plus que d'un droit citoyen plein et entier. Toutefois l'ancrage politique des chrétiens n'est pas monolithique, les chrétiens des tribus jordaniennes étant, par leur histoire, plus liés au pouvoir tandis que les chrétiens palestiniens peuvent être davantage liés aux partis de gauche.

Certes, ils connaissent les affres de l'insécurité régionale et l' " islamisme du quotidien », mais le pouvoir veille à contrôler les islamistes, notamment le Front de l'action islamique. Ainsi, les zones rurales plus loyales au régime sont proportionnellement davantage représentées à l'Assemblée que les villes où l'électorat des Frères est le plus élevé. Le pouvoir offre ainsi aux chrétiens une garantie de sécurité politique et de reconnaissance. La famille royale est particulièrement vigilante là-dessus : la femme du roi, Rania, est chrétienne et l'environnement du roi est très impliqué dans le dialogue islamo-chrétien ${ }^{10}$.

Au Liban, la situation des chrétiens n'a rien à voir avec celle de leurs coreligionnaires d'Irak, de Syrie et de Palestine. Ils continuent de prendre une part très active sur le plan social, éducatif et économique. L'université Saint-Joseph est également le grand centre universitaire du

8 Mediapart, 22 septembre 2014.

9 Cet accord n'est pas une reconnaissance de la Palestine, que le Vatican avait déjà reconnue, mais un accord sur le droit de l'Église catholique dans les territoires occupés.

10 En novembre 2011, le Forum islamo-catholique s'est tenu en Jordanie sur le thème : « Raison, foi et personne humaine, perspectives chrétiennes et musulmanes ». Cette rencontre a réuni des personnalités venues de dix-huit pays. 
dialogue islamo-chrétien dans la région. Sur le plan politique, les maronites, fondateurs de la république du Liban, en exercent la fonction présidentielle. Avant la guerre civile (1975-1990), ils ont ainsi pu se prévaloir d'une certaine prééminence politique, avec les communautés druze et sunnite, sur les chiites. Le rééquilibrage communautaire des accords de Taëf en 1989 a été d'autant plus mal vécu par les chrétiens ${ }^{11}$ que la mainmise syrienne qui les a suivis a évincé certains de leurs représentants de premier plan tandis que des chrétiens prosyriens, peu populaires, ont été promus.

Le départ des forces syriennes en avril 2005 a reconfiguré en partie l'échiquier politique libanais mais là encore selon des lignes communautaires, notamment chez les musulmans : la coalition du 14 Mars $^{12}$ accueille un Courant du futur à forte composition sunnite, tandis que le bloc du 8 Mars compte l'essentiel des représentants chiites (Amal et Hezbollah). De leur côté, les chrétiens se sont retrouvés de part et d'autre de l'échiquier politique, à l'instar de deux anciennes figures de la guerre civile: Samir Geagea, lié au bloc du 14 Mars soutenu par l'Occident et les pays du Golfe, et Michel Aoun, associé au Hezbollah au sein du bloc du 8 Mars dont l'Iran est de fait le parrain; au sein de ce bloc, citons également Sleiman Frangié, le chef des Marada, une formation politique qui s'engagea en tant que milice pendant la guerre civile libanaise. En soi, cette situation non monolithique des chrétiens tend à faire penser qu'ils sont les moins «recommunautarisés » du Liban, au moins sur le plan politique. Mais les positionnements des leaders politiques révèlent-t-il vraiment les sentiments des Libanais fatigués par tant de jeux de pouvoir sans avancées sur le plan des politiques publiques? Les luttes entre leaders chrétiens, avec en arrière-plan une vacance de la fonction présidentielle, intéressent-elles réellement la communauté chrétienne? Cependant il y a là un paradoxe car, même si les chrétiens libanais (et au-delà) relativisent la portée du pouvoir du président maronite, cette vacance politique encore d'actualité en ce début d'année 2016, et qui est liée à la mésentente chrétienne mais aussi à celle qui prévaut entre parrains du Liban (l'Arabie saoudite et l'Iran), n'est pas faite pour les rassurer dans un moment de grande instabilité régionale. Le revirement inattendu et récent de Samir Geagea, qui s'est dit prêt à soutenir la candidature de Michel Aoun, est-il de nature à les apaiser ?

11 Les chrétiens ont perdu une certaine suprématie politique du fait du rééquilibrage des sièges au Parlement et de l'affaiblissement des prérogatives du président de la République maronite (il a perdu le rôle de chef de l'exécutif au profit du président du Conseil des ministres et il ne peut plus révoquer le Parlement.)

12 Ce courant accueille les partis qui avaient organisé la manifestation antisyrienne le 14 mars 2005, soit quelques semaines avant le départ des forces syriennes sous pression internationale. Le bloc du 8 Mars regroupe les partis prosyriens qui avaient remercié la Syrie avant son départ annoncé. 


\section{Conclusion}

Dans cet article, si nous avons considéré les Arabes chrétiens comme le prisme des dysfonctionnements et échecs du monde arabe, il va sans dire qu'ils ne sont pas, loin s'en faut, les seuls perdants de cette mauvaise donne : c'est l'ensemble des populations du monde arabe, dont les sunnites aussi à l'instar de Syriens de Syrie, qui souffre de l'islamisme radical, du maintien des régimes autoritaires et corrompus, de l'absence d'offres politiques crédibles et de l'état de guerre. Ce qui, en revanche, est plus spécifique de la situation des Arabes chrétiens, c'est le scénario de leur disparition, du moins comme en Syrie, Irak et Palestine, où l'empreinte chrétienne a été forte. Au-delà du drame des départs forcés ou plus encore des enlèvements et assassinats, c'est la liquidation d'un certain métissage culturel qui ferait gravement défaut à ces pays.

Mais on connaît aussi les conditions de leur maintien dans le monde arabe : la lutte contre l'islamisme radical, l'apaisement de la fitna islamique qui, par ricochet, affecte les chrétiens, le règlement du conflit israélo-palestinien, l'avènement de politiques économiques inclusives, etc. Une autre évolution serait indispensable : la recherche d'une formule politique où ils pourraient demeurer de plain-pied dans le corps politique des pays arabes. Cette perspective avait été revendiquée sous une forme et dans un contexte singulier par l'évêque irakien Shlimoun Warduni lors de la construction de la nouvelle Constitution de son pays : «Nous voulons un État démocratique, civil, pluraliste, fédéral, qui sépare la religion et l'État, c'est-à-dire la nonpolitisation de la religion et la non-religiosation de la politique. » Mais pour que ce type de construction devienne opérant, il faut encore du temps d'autant plus que les conflits retardent les réformes en profondeur. Dans certains pays de la région, les Arabes chrétiens seront-ils encore là lorsqu'elles verront le jour ?

\section{Bibliographie}

BARRENADA I. (2013), «Les Palestiniens de citoyenneté israélienne, vingt ans après Oslo », Confluences Méditerranée, $\mathrm{n}^{\circ} 86$.

BENDÉLAC J. (2008), Les Arabes israéliens, entre intégration et rupture, Paris, Autrement. BLANC P. et CHAGNOLLAUD J.-P. (2014), Violence et politique au Moyen-Orient, Paris, les Presses de Sciences Po.

CARRÉ O. (2004), Mystique et politique, le Coran des islamistes. Lecture du Coran par Sayyid Qutb, Frère musulman radical (1906-1966), Paris, Cerf. 
CORM G. (2015), Pensée et politique dans le monde arabe, Paris, La Découverte.

COURBAGE Y. et B OUSTANI R. (2008-2009), «Évolution démographique communautaire au Liban et ses conséquences », Travaux et jours, revue de 1'USJ, automne 2008-hiver 2009, p. 129-148.

COURBAGE Y. et FARGUES P. (1992), Chrétiens et juifs dans l'islam arabe et turc, Paris, Fayard, p. 184-185.

DAGHER C. (2002), Le Défi du Liban d'après-guerre, Paris, L’Harmattan.

FADDOUL J. (2007) « La difficile mais nécessaire arabité des maronites », Maghreb-Machrek, $\mathrm{n}^{\circ} 192, \mathrm{p} \cdot 71-85$

FIERRO M. (2009), «Comment les musulmans d'Al-Andalus voient les autres religions », in Chrétiens face à l'islam, Paris, Bayard

FLEYFEL A. (2011), La Théologie contextuelle arabe. Modèle libanais, Paris, L’Harmattan.

GUIRGUIS L. (2012), Les Coptes d'Égypte, violences communautaires et transformations politiques (2005-2012), Paris, Karthala.

KASSIR S. (2004), Considérations sur le malheur arabe, Arles, Actes Sud.

KEPEL G. et MILELLI J.-P. (2005), Al-Qaïda dans le texte, Paris, coll. « Proche-Orient ».

KHADER B. (1984), La Question agraire dans les pays arabes, Louvain, CIACO éditeur.

OTAYEK R. (2013), «Pluralisme culturel et régime(s) politique(s). Un essai de comparaison Afrique/monde arabe », Revue internationale comparée, vol. 20, n², p. 101-123.

DE PLANHOL X. (1968), Les Fondements géographiques de l'histoire de l'islam, Paris, Flammarion.

QOTB S. (2000), « The America I have seen », in ABD EL-MALEK K. et EL KAHLA M. (dir.), America in an Arab Mirror. Images of America in Arabic Travel Literature, New York, St. Martin's Press.

SENIGUER H. (2015), «Une terreur sacrée ? La violence à l'heure des crises du MoyenOrient », Confluences Méditerranée, $\mathrm{n}^{\circ} 94$.

URVOY D. (2010), «Entre échanges et conflit, le mythe andalou », Le Monde la Bible, horssérie. 